University of Wollongong

Research Online

1994

\title{
Legionnaires' disease outbreak, Fairfield 1992: public health aspects
}

\author{
Michael Levy \\ New South Wales Health \\ Victoria J. Westley-Wise \\ University of Wollongong, victoria@uow.edu.au \\ Charles Blumer \\ New South Wales Health \\ Michael Frommer \\ New South Wales Health \\ George Rubin \\ New South Wales Health
}

See next page for additional authors

Follow this and additional works at: https://ro.uow.edu.au/ahsri

Research Online is the open access institutional repository for the University of Wollongong. For further information contact the UOW Library: research-pubs@uow.edu.au 


\title{
Legionnaires' disease outbreak, Fairfield 1992: public health aspects
}

\begin{abstract}
An investigation of an outbreak of Legionnaires' disease in 1992 in Fairfield, a municipality of Sydney, was carried out to determine the source of the outbreak. Cases of Legionnaires' disease with onset of symptoms between 11 and 20 April 1992 were included. Definite cases were individuals with a history consistent with Legionnaires' disease, confirmed by direct fluorescent antibody testing plus serology or culture. There were two control groups: patients admitted to the same hospital as the cases, matched for age and sex, and patients admitted to hospital with a presumptive diagnosis of Legionnaires' disease, in whom the diagnosis was subsequently excluded. There were 26 definite cases with onset of symptoms between 11 and 20 April 1992. Six (23 per cent) died. Twenty-two cases (85 per cent) reported visiting the Fairfield business district during the ten days prior to the onset of symptoms. They were 20 times more likely to have visited Fairfield than were matched controls. Matching of Legionella pneumophila serogroup 1 from environmental and clinical samples was achieved by cytogenetic fingerprinting. Fourteen cases were linked to a single environmental sample. The epidemiological findings were consistent with a point source of Legionella in the Fairfield business district. It is most likely that the exposure occurred on 10 April 1992.
\end{abstract}

\section{Keywords}

legionnaires', 1992:, fairfield, public, outbreak, health, aspects, disease

\section{Publication Details}

M. Levy, V. Westley-Wise, C. Blumer, M. Frommer, G. Rubin, D. Lyle, J. Brown \& G. Stewart, "Legionnaires' disease outbreak, Fairfield 1992: public health aspects", Australian Journal of Public Health 182 (1994) 137-143.

\section{Authors}

Michael Levy, Victoria J. Westley-Wise, Charles Blumer, Michael Frommer, George Rubin, David Lyle, John Brown, and Greg Stewart 


\title{
Legionnaires' disease outbreak, Fairfield 1992: public health aspects
}

\author{
Michael Levy, Victoria Westley-Wise, Charles Blumer, Michael Frommer, \\ George Rubin and David Lyle \\ Epidemiology and Health Services Evaluation Branch, New South Wales Health Department
}

\author{
John Brown and Greg Stewart \\ South Western Sydney Area Health Service Public Health Unit
}

\begin{abstract}
An investigation of an outbreak of Legionnaires' disease in 1992 in Fairfield, a municipality of Sydney, was carried out to determine the source of the outbreak. Cases of Legionnaires' disease with onset of symptoms between 11 and 20 April 1992 were included. Definite cases were individuals with a history consistent with Legionnaires' disease, confirmed by direct fluorescent antibody testing plus serology or culture. There were two control groups: patients admitted to the same hospital as the cases, matched for age and sex, and patients admitted to hospital with a presumptive diagnosis of Legionnaires' disease, in whom the diagnosis was subsequently excluded. There were 26 definite cases with onset of symptoms between 11 and 20 April 1992. Six (23 per cent) died. Twenty-two cases (85 per cent) reported visiting the Fairfield business district during the ten days prior to the onset of symptoms. They were 20 times more likely to have visited Fairfield than were matched controls. Matching of Legiomella pmeumophila serogroup 1 from environmental and clinical samples was achieved by cytogenetic fingerprinting. Fourteen cases were linked to a single environmental sample. The epidemiological findings were consistent with a point source of Legionella in the Fairfield business district. It is most likely that the exposure occurred on 10 April 1992. (Aust J Public Health 1994; 18: 137-43)
\end{abstract}

A cluster of cases of Legionnaires' disease occurred in Fairfield in April 1992. Fairfield is a municipality of Sydney situated $23 \mathrm{~km}$ southwest of the Sydney central business district.

An investigation was initiated on 21 April 1992 following the notification to South Western Sydney Public Health Unit of two cases in Liverpool Hospital over the Easter long weekend (17-20 April 1992), one of whom had died. Following standard protocols for responding to a notification of Legionnaires' disease,' the unit identified 15 patients who had presented to the Fairfield Hospital Accident and Emergency Department with atypical pneumonia over the Easter long weekend. On 22 April a second death from Legionnaires' disease in Liverpool Hospital was notified.

\section{Methods}

Case finding of all forms of atypical pneumonia presenting to New South Wales (NSW) hospitals was carried out by Public Health Unit staff by contacting each hospital in the Public Health Unit's respective Area or Region.

We calculated the background incidence of Legionnaires' disease and other forms of atypical pneumonia in South Western Sydney and New South Wales by analysing the NSW Inpatient Statistics Collection on hospital separations for Legionnaires' disease (ICD9-CM 482.8, pneumonia due to other specified bacteria) and atypical pneumonia (ICD9--CM 483, pneumonia due to other specified organisms).

Correspondence to Dr Michael I.evy, Epidemiology and Health Services Evaluation Branch, NSW Health Department, 73 Miller Street, North Sydney, NSW 2060. Fax (02) 3919232.
Australian Bureau of Statistics resident population estimates for NSW were used to calculate rates. ${ }^{2}$

Public health officers, public health nurses and staff from the Epidemiology Branch administered a structured questionnaire to patients with atypical pneumonia to determine potential sources of exposure. Relatives of patients were interviewed if the patients were deceased or too ill to be interviewed. The investigation was confined to definite and probable Legionnaires' disease cases admitted to a hospital in South Western Sydney or Western Sydney, according to the following case definitions:

1. Definite case: clinical history consistent with Legionnaires' disease, and positive direct fluorescent antibody test serology or culture for Legionella pmeumophila serogroup 1 .

2. Probable case: all of the following criteria had to be met:

- illness of less than two weeks' duration

- aged more than 20 years

- no alternative microbiological diagnosis

- chest x-ray signs of pneumonia (infiltrate and/or opacity)

- laboratory evidence of hypoxia $\left(\mathrm{p}_{2} \mathrm{O}_{2}<85 \mathrm{~mm}\right)$.

3. In addition, two or more of the following criteria had to be met:

- gastrointestinal symptoms

- central nervous system symptoms or signs

- history of heavy smoking

- immunosuppression

- myalgia

- prostration

- biochemical abnormalities (hyponatraemia, raised creatinine kinase, abnormal liver function tests or abnormal renal function tests).

We established a computerised database for cases and controls with a separate database for environmental sampling data, using Epilnfo version $5.01 \mathrm{~b} .{ }^{x}$ 
Conditional odds ratios (OR) and 95 per cent confidence intervals (CI) were calculated using the MULTI.R logistic regression program. ${ }^{4}$

\section{Sources of Legionella infection}

We investigated potential sources of Legionella contamination by:

- environmental surveys and water sampling at potential sites of exposure identified from case interviews

- case-control investigations to compare the likelihood that cases and controls had been exposed to these sites, and

- examination of Legiomella from sputum or bronchial secretions and environmental samples.

Environmental samples were initially taken from sites suggested by exposure histories given by cases. Subsequently, the sampling was extended to all water-cooled air conditioning systems in the Fairfield business district and then throughout South Western Sydney. Water samples were collected over the period 23 April to 1 May from 203 water-based systems: 200 cooling towers from water-based air conditioning systems, and three fountains in public areas.

\section{Laboratory methods}

Clinical samples were analysed using direct fluorescent antibody testing of induced sputum, serology and culture. ${ }^{5}$ Sputum samples with positive cultures for $L$. pmeumophila serogroup 1 were analysed by cytogenetic fingerprinting, using a method enhancing restriction fragment length polymorphism. ${ }^{6}$

Cytogenetic fingerprinting was undertaken on all culture-positive environmental samples with more than 100 colony-forming units (CFU) per ml of $L$. pmeumophila serogroup 1 coming from sites visited by cases.

\section{Case-control investigations}

To investigate hypotheses on times and places of patient exposure to Legionella we conducted a casecontrol study with two control groups:

1. a primary matched control group $(n=45)$. One or two controls were individually matched on sex, age (within five years), hospital of admission, and date of admission (within two days following the admission of the case) for each case. If a control could not be identified using these criteria, a selection was made from the hospital admission list within two days prior to the admission of the case and using the same matching criteria, and

2. a secondary unmatched control group $(n=75)$ comprising 'probable Legionnaires' disease' cases admitted to hospital, where Legionnaires' disease was subsequently excluded when neither direct fluorescent antibody test, serology or culture confirmed the diagnosis. This control group was used for unmatched analyses.

The Legionnaires' disease cases and controls were asked to report on locations that they visited in the ten days prior to the onset of their symptoms.

We used matched and unmatched analyses of the case-control data. A hierarchical approach was taken, starting with broad geographical locations (suburbs), and then focusing on specific sites, guided
Table 1: Hospital separations for legionnaires' disease and atypical pneumonia in South Western Sydney (SWS) and New South Wales (NSW), 1989-90 and 1990-91

\begin{tabular}{lcccc}
\hline & \multicolumn{2}{c}{$1989-90$} & \multicolumn{2}{c}{$1990-91$} \\
& SWS & NSW & SWS & NSW \\
\hline Legionnaires' disease & 7 & 79 & 2 & 69 \\
Atypical pneumonia & 34 & 416 & 29 & 348 \\
\hline
\end{tabular}

Source: NSW Inpatient Statistics Collection, NSW Health Department

by the results of the initial Legionnaires' disease case interviews. The following refers to the analyses using the matched controls.

\section{Results}

Early reports from hospitals around the state suggested that the initial cases of Legionnaires' disease and other forms of atypical pneumonia comprised an excessive occurrence. By 24 April, statewide case-finding had revealed 110 definite or probable cases of Legionnaires' disease with dates of onset between 11 April and 20 April.

Data from the Inpatient Statistics Collection for Legionnaires' disease and other forms of atypical pneumonia in 1989-90 and 1990-9l were as shown in Table 1.

We determined from these data that the occurrence of Legionnaires' disease in South Western Sydney in April 1992 was excessive. Subsequently the investigation was confined to Legionnaires' disease. A total of 183 questionnaires was administered between 22 April and 4 May.

Twenty-six definite cases of Legionnaires' disease were identified with dates of onset between 11 and 20 April, the modal dates being 13 and 15 April (Figure 1). Four ( 15 per cent) of the cases were females, and 22 (85 per cent) were males. The ages ranged from 24 to 89 years, with a mean age of 57.7 years (standard deviation (SD) 18.8 years). The mean age for males was 55.2 years (SD 14.7 years), and the four females were aged $62,67,72$ and 89 years. There were six deaths, representing a case fatality rate of 23 per cent. The dates of onset of Legionnaires' disease in the patients who died were 13 April (three cases), 15 April (two) and 16 April (one). All six deaths were male. The mean age of the deceased was 54.0 years (range 42 to 73 years, SD 14.0 years).

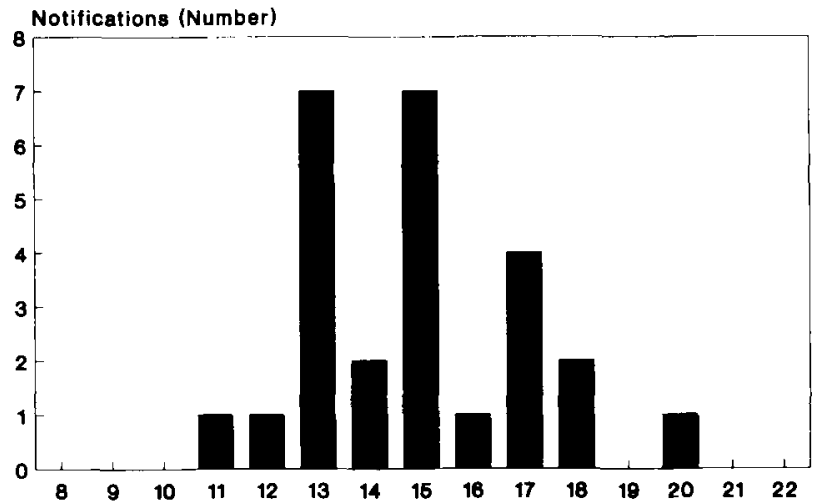

Figure 1: Epidemic curve for Legionnaires' disease, Foirfield, April 1992. Source: Infectious Diseases Surveillance System. 
Table 2: Frequency of Legionnella colonisation levels (colony-forming units per $\mathrm{ml}$ ) in samples from water-based systems, by Local Government Area, 23 April to 1 May 1992

\begin{tabular}{lrrrr}
\hline & $<10$ & $10-100$ & $>100$ & Total \\
\hline Local Government Area & & & & \\
Fairfield & 78 & 5 & 7 & 90 \\
Liverpool & 28 & 7 & 4 & 39 \\
Bankstown & 11 & - & 6 & 17 \\
Camden & 9 & 1 & - & 10 \\
Campbelltown & 36 & 3 & 8 & 47 \\
Total & 162 & 16 & 25 & 203 \\
\hline
\end{tabular}

\section{Case-control investigations}

The case--control analysis confirmed that the source of Legionella was likely to have been in Fairfield. Cases were 20 times more likely than controls to have visited Fairfield (OR 19.9, CI 2.6 to 153). No statistically significant associations were found for visiting other suburbs.

Twenty-two of the 26 cases ( 85 per cent) and 11 of the 45 matched controls ( 24 per cent) reported visiting sites in the Fairfield business district during the ten days prior to the onset of their symptoms (OR 17.0, CI 4.8 to 60.0). Matching was dissolved for the calculation of this $O R$ as a matched odds ratio could not be computed due to the lack of case-control pairs in which both the case and the control had not visited the Fairfield business district.

Compared with matched controls, cases were 22 times more likely to report visiting sites on Smart Street (OR 22.3, CI 2.90 to 170.0), and nine times more likely to have visited sites on Ware Street (OR 8.9 , CI 2.0 to 40.0). Results from the analyses using the unmatched controls were consistent with those of the matched controls (Smart Street: OR 17.6, CI 5.2 to 62.9, Ware Street: OR 13.4, CI 4.0 to 46.7).

Small numbers of cases and controls reported visiting sites outside the Fairfield business district, but we found no significant associations with Legionnaires' disease.

\section{Envirommental surveys and water sampling}

One hundred and sixty-two (80 per cent) of the systems tested had fewer than ten CFUs per ml (Table 2 ), indicating that maintenance practices were effective at the time of sampling. ${ }^{7}$ Fifteen samples had 10 to $100 \mathrm{CFUs}$ per $\mathrm{ml}$, indicating that maintenance practices may not have been satisfactory. Twenty-five systems had over 100 CFUs per $\mathrm{ml}$, the level specified as potentially hazardous. Fifteen of these systems yielded positive cultures for Legionella pmeumophila serogroup 1.

Three systems in the Fairfield business district were found to be contaminated with Legiomella species, two with $L$. pneumophila serogroup 1 .

Relationship of serogroups and cytogenetic 'fingerprinting' results for Legionella from patients and environmental samples

Water samples from 11 systems in South Western Sydney culturing $L$. pmeumophila serogroup 1 with more than $100 \mathrm{CFUs}$ per $\mathrm{ml}$ and sputum samples from 16 of 17 Legionnaires' disease cases with positive cultures were tested genetically (the seventeenth culture was not successfully transported to the testing laboratory). The cytogenetic fingerprinting results for the organisms from the sputum samples of 14 of the 16 cases ( 88 per cent) matched the cytogenetic fingerprint of organisms cultured in the water sample taken from one restaurant cooling tower on 23 April 1992. There was no match between the 14 sputum samples with the fingerprint of organisms from other environmental samples taken during the investigation. Cytogenetic fingerprinting revealed that sputum samples from the remaining two cases were unrelated to any of the organisms cultured from environmental samples.

\section{Weather readings}

Weather conditions are monitored daily between 6 a.m. and 3 p.m. at Bankstown Airport, $6 \mathrm{~km}$ southeast of Fairfield. A summary of weather conditions between 8 and 13 April is presented in Table 3.

\section{Discussion}

Legionnaires' disease

Legionnaires' disease is an acute bacterial pneumonia caused by $L$. pneumophila and related Legionella species. The incubation period of the disease is two to ten days. The modal incubation period is five to six days.

Diagnosis depends upon antigen detection by direct fluorescent antibody test, sputum culture and serology. The advantage of the antibody test is its rapidity and specificity; however, the sensitivity of the test is relatively low, so some false negative results are

Table 3: Weather conditions, Bankstown Airport, 8 to 13 April 1992

\begin{tabular}{|c|c|c|c|}
\hline Date & Temperature range & Wind direction & General comments \\
\hline $\begin{array}{l}8 \text { April } \\
9 \text { April } \\
10 \text { April }\end{array}$ & $\begin{array}{l}15-18^{\circ} \mathrm{C} \\
12-19^{\circ} \mathrm{C} \\
11-21^{\circ} \mathrm{C}\end{array}$ & $\begin{array}{l}\text { Southerly } \\
\text { South, southeasterly } \\
\text { Southeasterly }\end{array}$ & Rain showers to the east at midday \\
\hline 11 April & $12-24^{\circ} \mathrm{C}$ & Northerly & $\begin{array}{l}\text { High barometric pressure, } 2 \text { millibar drop between } 12 \text { noon and } 1 \\
\text { p.m. (1027 to } 1025)\end{array}$ \\
\hline 12 April & 13 to $24^{\circ} \mathrm{C}$ & $\begin{array}{l}\text { Calm winds in the morning, } \\
\text { northerly in the afternoon }\end{array}$ & Fog and mist in the morning gave way to smoke haze later in the day \\
\hline 13 April & 12 to $24^{\circ} \mathrm{C}$ & Calm winds all day & Fog and mist in the morning gave way to smoke haze later in the day \\
\hline
\end{tabular}


inevitable. There can be a delay of up to three months until the results of the most sensitive confirmatory investigations - culture and convalescent serologyare available. For these reasons, clinical diagnoses and notifications of Legionnaires' disease are often delayed. Thus, the clinical and microbiological characteristics of Legionnaires' disease may lead to difficulties in mounting a timely public health response to Legionnaires' disease outbreaks.

Legionellae are ubiquitous contaminants of reticulated water. They are present in the mains water supply in small numbers but proliferate in warm water at temperatures below $50^{\circ} \mathrm{C}$, and in areas of stagnation and sludge formation, in the presence of amoebae and other bacteria. They can achieve high numbers in warm water systems of large buildings. ${ }^{8.4}$ Formation of aerosols from the agitation of contaminated water, which are then inhaled, is a major mode of spread of Legionellae.

Safe levels of Legionellae in the cooling towers of air conditioning systems have been defined. ${ }^{\top}$ As for clinical specimens, a combination of culture and antigen detection by immunofluorescence offers the best method for identifying Legionellae in environmental samples.

\section{Legiomnaires' disease in New South Wales}

The outbreak of Legionnaires' disease in South Western Sydney in April 1992 was the largest in NSW since an outbreak at Wollongong in April 1987. In that outbreak, 44 cases of Legionnaires' disease (including nine deaths) were reported. ${ }^{10}$ There was strong evidence linking the outbreak to the Wollongong Shopping Mall. Monoclonal antibody test results from seven cases and from cooling tower water from a building in the mall were of the identical serotype. Cytogenic fingerprinting was undertaken by the Centers for Disease Control, Atlanta. However, no analytic epidemiological study was performed. The public health response involved cleaning all cooling towers in the Wollongong district and closing down the air conditioning system of the shopping mall.

In April 1989, 12 confirmed or probable cases of Legionnaires' disease in residents of the western suburbs of Sydney were investigated; seven of the cases reported visiting the Merrylands Bowling Club." A case-control study was not performed because of the relatively small number of cases. In the absence of an analytic study and because the subtypes of available clinical and environmental specimens were different, no conclusions could be drawn as to whether the Merrylands Bowling Club was the source of the infection.

It is notable that the three Legionnaires' disease outbreaks in NSW have occurred in April. A review of Legionnaires' disease notifications in South Australia noted a summer predominance. ${ }^{12}$ The only recorded outbreak in New Zealand occurred in February. ${ }^{13}$ A report from Scotland noted that Legionnaires' disease not associated with travel had a summer-autumn peak. ${ }^{14}$ The frequency of outbreaks of Legionnaires' disease in autumn is reviewed extensively elsewhere. ${ }^{15}$

A possible explanation for the April outbreaks in NSW is fluctuations in ambient temperatures resulting in air conditioning plants being cycled on and off,

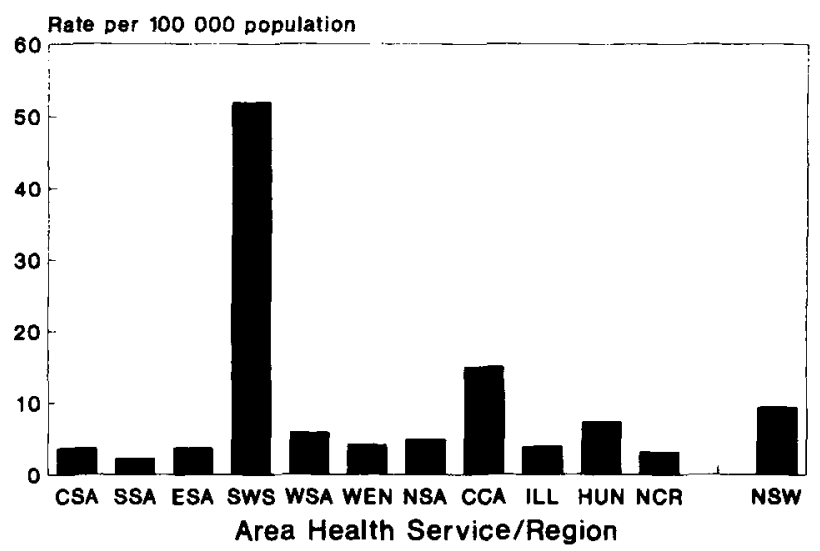

Figure 2: Legionnaires' disease rate, April 1992, by Area Health Service or Region. SWS = South Western Sydney Area. Source: Infectious Diseoses Surveillance System.

with the result that the build-up of Legionellae was discharged into the environment. The weather pattern between 8 and 13 April may have led to air conditioning plants being cycled off around 8 April, and cycled on again from 10 April; on that day, night temperatures in the area dropped to $11^{\circ} \mathrm{C}$, with the 1 p.m. temperature being $21^{\circ} \mathrm{C}$ at Bankstown Airport.

During 1992, 103 notifications for Legionnaires' disease were received by the NSW Health Department, a rate of 1.75 cases per 100000 population. The notification rate for NSW for April was 9.35 per 100000 population, with South Western Sydney reporting a rate of 51.96 per 100000 (Figure 2).

The weekly distribution of notifications for 1992 (Figure 3) reveals that even excluding those cases known to be associated with the Fairfield cluster, weeks 15 to 17 had unusually high numbers of notifications compared to other weeks. This may have been a result of:

- improved case ascertainment due to heightened awareness of Legionnaires' disease by clinicians,

- conditions favouring Legionnaires' disease throughout the state,

- further cases of Legionnaires' disease exposed in Fairfield, but not linked to the cluster in our investigation, and

- further clusters of Legionnaires' disease which were not identified.

Reports from the United Kingdom have stated that apparent sporadic cases of Legionnaires' disease may

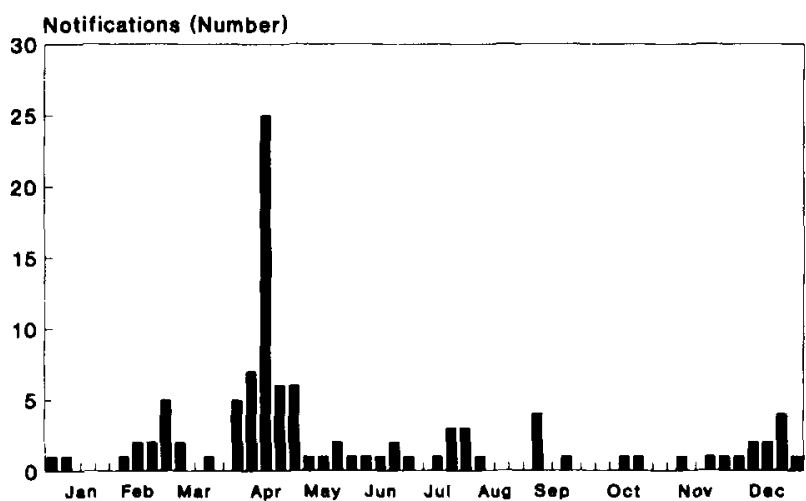

Figure 3: Legionnaires' disease notifications by week of onset. New South Wales, 1992. Source: Infectious Diseases Surveillance System. 
be linked in space and time. ${ }^{16}$ We confirmed this in the current outbreak, when two apparently unrelated cases of Legionnaires' disease were notified to the Public Health Unit. Active surveillance by the unit revealed 15 further cases in South Western Sydney.

\section{Public health action}

The primary aims of the investigation were:

- to determine whether the outbreak emanated from a propagated (continuing) or point source exposure, to identify the most probable source(s), and

- to assess and manage any continuing risk.

Within 24 hours of notification of the outbreak of Legionnaires' disease, preliminary results from this investigation indicated that a point source was most likely, and that the epidemic was centred in South Western Sydney.

Early in the investigation, the Director of Public Health for the area directed the occupiers of premises with water-cooled air conditioning systems which had not been serviced in the previous four weeks to immediately shut the systems down for cleaning procedures as outlined in the NSW code of practice for the control of Legionnaires' disease. ${ }^{7}$ Where possible, samples were taken prior to shutdown, although this was rarely possible

Subsequently, all water-based air conditioning systems in South Western Sydney found to be contaminated with Legionellae at levels greater than $100 \mathrm{CFU}$ per $\mathrm{ml}$ were decontaminated. Public health unit and local government environmental health officers were guided on appropriate steps to be taken by the NSW code of practice for the control of Legionnaires' disease. ${ }^{7}$

The three contaminated systems in the Fairfield business district were decontaminated and no Legiomellae were detected in subsequent water samples. On 28 and 29 April, public health unit and local council environmental health officers confirmed that all other contaminated water-based systems had been resampled and no further contaminated systems were found.

No new epidemic-related cases of Legionnaires' disease were notified after 25 April. On 29 April a media release was issued by the Chief $\mathrm{Health}$ Officer, informing the public that the epidemic appeared to have been controlled.

The epidemic curve shows an abrupt onset. The cessation of the outbreak within the duration of the maximum incubation period of Legionnaires' disease is consistent with exposure to a single point source. Considering the two to ten days incubation period of Legionnaires' disease and the dates of onset of the cases, it is probable that exposure occurred between 9 and 11 April 1992. This is reinforced by the history of one person who resided over 50 kilometres from Fairfield, but visited the district on one occasion only-midday on 10 April.

The nonspecific clinical symptoms and inevitable delays in confirming the clinical diagnosis emphasise the need for, and the difficulty in devising, a case definition during a Legionnaires' disease outbreak investigation. The clinical case definition developed during this investigation should facilitate future Legionnaires' disease investigations. However, in this instance, more than 100 people were initially investigated statewide before the strict case definition was applied. In retrospect, use of the stricter case definition from the outset could have improved the efficiency of the response. However, until the nature of the epidemic was defined and the possibility of a concurrent outbreak of other forms of atypical pneumonia was rejected, a wide-ranging initial approach to case ascertainment was undertaken.

Consistency of the epidemiological, microbiological and cytogenetic fingerprinting data

In all 17 culture-positive cases, the organism was $L$. pmeumophila serogroup 1. Cytogenetic fingerprinting for 14 of the samples was of the identical organism. This particular fingerprint was found in only one environmental sample tested, that taken from a restaurant cooling tower in Smart Street. Thirteen of the 14 (93 per cent) were from cases who reported entering premises in Smart Street or Ware Street, Fairfield. Neither of the two remaining culturepositive cases reported visiting the Fairfield business district.

Epidemiological evidence cannot definitively establish the cause of an epidemic, but it can provide an indication of a likely source within the time constraints dictated by the urgent requirement for public health action. However, epidemiological methods have limitations. In the case-control analysis, cases' reports of the places which they had recently visited were compared with controls' reports. Data related to places visited were limited by problems of recall, particularly for cases and controls who were severely ill; in some instances the data were obtained from relatives.

Case-cont rol methodology has been used successfully to demonstrate an association between Legionnaires' disease and specific buildings during a prolonged Legionnaires' disease outbreak in Gloucester in the United Kingdom in 1986. ${ }^{17}$

Evidence from hospital-based case-control studies is considered inferior to that from population-based case-control studies. However, given the need to obtain a rapid analysis, the use of hospital controls was the most practical option in this investigation. The evidence from this study was supported by the use of a second (nonmatched) control series, which gave consistent conclusions. The nonmatched controls originated as possible Legionnaires' disease cases who turned out not to have I egionnaires' disease, but confirmation of their diagnostic status was unavailable for a few weeks. Therefore, while the second control series could not be used in initial hypothesis-testing, it provided confirmation of the early analytic findings.

Subjects' reports of contacts with particular locations are only proxies for actual exposures. Almost without exception, when cases or controls visited Fairfield, they visited several establishments. For this reason it is not surprising that, while the case-control results clearly link the outbreak with the Fairfield business district, the analyses also reveal significant associations between Legionnaires' disease and more than one establishment within the business district. Therefore, from the case-control analyses no single location was identified as the sole source of exposure. 
In the majority of documented Legionnaires' disease outbreaks, the Legionellae have emanated from a point source. If the organism proliferates in a cooling tower, it may be dispersed into the outdoor environment as an aerosol, which may then be inhaled by people in the vicinity. Alternatively, the organism may be drawn into an air conditioning system's air intake and swept indoors with the conditioned air. ${ }^{18}$

Where an indoor exposure is suspected, a single site is likely to be connected with a large proportion of cases. ${ }^{19} \mathrm{~A}$ similar sized outbreak, with similar ORs, was linked to indoor contamination by a mist machine. ${ }^{20}$ In that outbreak, employees of a particular store were more likely to have contracted Legionnaires' disease than controls.

In the present outbreak, no such pattern emerged; no more than 52 per cent of cases had visited any individual site in the Fairfield business district. This suggested outdoor exposures. Furthermore, groups of sites along each of the two major thoroughfares (Ware Street and Smart Street) appeared to be connected to a large proportion of cases. While this may support the hypothesis of an outdoor exposure, cases and controls were not questioned whether they actually entered specific sites or merely walked around the district.

Conclusive evidence implicating a single point source depends upon:

- the existence of a strong epidemiological association between disease and a postulated source,

- lack of association between disease occurrence and other sources, and

- the isolation of the same organism from both clinical and environmental specimens.

During the outbreak response it was not feasible to wait for conclusive evidence before adopting measures judged likely to be effective in minimising any continuing risk. This public health imperative led to a very early decision that all premises in Fairfield with relevant types of air conditioning systems carry out maintenance on cooling towers, or close down the systems. The decision was made before water samples could be taken from many of the cooling systems. Thus, many of the samples were collected after cleaning, and this may account for the small number of positive samples in the Fairfield business district. Legionella pneumophila serogroup 1 was isolated from only two of the many establishments which were visited by the Legionnaires' disease cases. Verifying links between these establishments and the cases of Legionnaires' disease was not possible until the results of cytogenetic fingerprinting of clinical and environmental samples became available.

The results from cytogenetic fingerprinting were crucial to the investigation because they excluded potential alternative sources of infection which could not otherwise be ruled out on the basis of epidemiological evidence.

\section{Conclusions}

Findings from the case-control study carried out as part of the investigation were consistent with exposure to a point source within the Fairfield business district, beginning between 9 and 11 April. One case had had a single opportunity for exposure, on 10 April. The epidemiological findings suggest a single point source of Legiomella exposure in the district, consistent with outdoor contamination originating in the vicinity of Smart or Ware Streets. Although the duration of the outbreak was brief, its manifestations were very serious; the case fatality rate was 23 per cent.

The justification for undertaking a major investigation may be questioned in hindsight, as the ability to affect the outcome of a point-source epidemic with onset days or even weeks after initiating the response is small, but at the time of initiating the outbreak response there had been limited experience in NSW in Legionnaires' disease outbreaks. The Wollongong outbreak had characteristics of a propagated outbreak, one with a prospect of containing the public threat during an outbreak response. That this turned out not to be the situation does not question carrying out this outbreak investigation, but raises the threshold for activating a major response in the future.

These concepts have been incorporated into revised editions of the NSW Health Department's Legionnaires' disease emergency management plan which stresses local response to locally identified clusters of Legionnaires' disease cases. ${ }^{21}$

That three clusters of Legionnaires' disease have been identified in April has led the NSW Health Department to issue warnings in March for air conditioner owners to be extra-vigilant about maintenance and cleaning standards in the ensuing weeks.

\section{Acknowledgments}

The authors wish to thank Professor Ian Webster, Director of Public Health South Western Sydney, Dr Sue Morey, NSW Chief Health Officer, and over 50 devoted NSW Health Department and Fairfield City Council officers who participated in the outbreak response.

\section{References}

1. Infectious diseases manual. Sydney: NSW Health Department, 1991 .

2. Age and sex distribution of the estimated resident population of statistical local areas: New South Wales. Cat. no. 3209.1. Canberra: Australian Bureau of Statistics, 1989.

3. Dean AG, Dean JA, Burton AH, Dicker RC. Epilnfo, Version 5.01 b: Stone Mountain, Georgie: USD Incorporated, 1991.

4. Campos-Filho N, Franco EL. A microcomputer program for multiple logistic regression by unconditional and conditional maximum likelihood methods. $A m J$ Epidemiol 1989; 129 ; 439-44.

5. Munro R, Neville S, Daley D, Mercer J. Microbiological aspects of an outbreak of Legionnaires' disease in South Western Sydney. Pathology 1994; 26: 48-51.

b. Saunders NA, Harrison TG, Haththotuwa A, Kachwalla N, Taylor AG. A method of typing strains of Legionella pmeumophila serogroup 1 by analysis of restriction fragment length polymorphisms. $J$ Med Microbiol 1990; 31: 45-55.

7. NSW code of practice for the control of Legionnaires' disease. Sydney: NSW Health Department, 1991.

8. Marrie TJ, Haldane D, Bezanson G, Peppard R. Each water outlet is a unique ecological niche for Legionella pneumophila. Epidemiol Infect 1992; 108: 261-70.

9 Bezanson C, Burbridge $S$, Haldane D, Yoell C, Marrie T. Diverse populations of Legionella pneumophila present in the water of geographically clustered institutions served by the same water reservoir. J Clin Microbiol 1992; 90: 570-6.

10. Christopher PJ, Noonan LM, Chiew R. Epidemic of Legionnaires' disease in Wollongong. Med J Aust 1987; 147: 127-8. 
11. Christley S, Rubin C, Christopher P. Legiomnaires' disease Westerm Sydny, NSW' Sydney: NSW Department of Health, 1989.

12. Cameron S, Roder D, Walker C, Feldheim J. Epidemiological characteristics of Legiomella infection in South Australia: implications for disease control. Aust $N Z J$ Med 1991; 21 : $6.5-70$.

13. Mitchell P, Chereshsky A, Haskell AJ, Brieseman MA l.egionellosis in New Zealand: first recorded outbreak. $N Z$ Med J 1991; 104: 275-6.

1 t. Bhopal RS, Fallon RJ. Seasonal variation of l.egionnaires disease in Scotland. J Infect 1991; 22: 153-60.

15. Bentham RH, Broadbent CR. A model for autumn outbreaks of legionnaires' disease associated with cooling towers, linked to system operation and size. Fipidemiol Infect 1993; $111: 287-95$
16. Bhopal RS, Diggle P, Rowlingson B. Pinpointing clusters of apparently sporadic cases of Legionnaires' disease. $B M J$ 1992; 304: 1022-7.

17. Hunt DA, Cartwright KA, Smith MC:, Middleton J, et al. An outbreak of Legionnaires disease in Gloucester. Fidemiol Infert 1991; 106: 133-41.

I.. Hoge CW, Breiman RF. Advances in the epidemiology and control of Legionella infections. Epidemiol Rev 1991; 13: $329-40$

19. O'Mahony MC, Stanwell-Smith RE, Tillett HE, Harper 1), et al. The Stafford outbreak of I egionnaires' disease. Epidemiol Infert 1990; 104: 361-80.

20) Mahonev FJ. Hoge CW, Farley TA, Barbaree JM, et al. Community-wide outbreak of Legionnaires' disease associated with a grocery store mist machine. J Infert Itis 1992; 165 736-9.

ㄴ. Legionnaires' disease emergency management plan. Sydney: NSW Health I)epartment, 1992

\title{
The behavioural epidemiology of weight control
}

\author{
David Crawford \\ National Centre for Epidemiology and Population Health, Canberra \\ Neville Owen \\ Department of Community Medicine, University of Adelaide
}

\begin{abstract}
Excess body weight is associated with increased health risk, but there are also risks to health related to weight-control practices. While representative population data are available on the anthropometry of body weight and its sociodemographic correlates in Australia, less is known about the population prevalence and correlates of weight-control behaviours. We examine the prevalence of overweight and obesity, and low body weight, in the Australian population, using data from studies in which height and weight have been objectively measured; we describe the sociodemographic correlates of overweight and obesity; we outline relevant aspects of Australian health-system and private-sector approaches to body weight control; and we describe the findings of Australian studies of weight-control practices and related beliefs and attitudes. These findings relate mainly to women, particularly younger women, and little is known about the weight-control practices of Australian men. We suggest research which may promote a better understanding of weight-control practices in the Australian population. (Aust J Public Health 1994; 18: 143-8)
\end{abstract}

$\mathrm{H}$ ealth authorities are concerned about excess body weight because it is a risk factor for a number of prevalent conditions, including hypertension, hyperlipidaemia, non-insulindependent diabetes mellitus, gall bladder disease and cardiovascular disease. ${ }^{1}$ Prospective studies have shown a J-shaped relationship between body weight and risk of premature mortality, with the highest levels of body weight being associated with greatly increased risk. Long-term prospective studies have shown that degree of overweight is an independent predictor of premature mortality. ${ }^{2}$ There are also concerns about low body weight, with individuals in this category being at increased risk." Overweight people are encouraged to reduce their weight, ${ }^{3}$ and those of low body weight to increase their weight to a 'healthier' level. ${ }^{3}$

In the context of these concerns and initiatives, an accurate and comprehensive understanding of the nature and extent of weight-control practices in the

Correspondence to Mr David Crawford, National Centre for Epidemiology and Population Health, The Australian National University, GPO Box 4, Canberra, ACT 2601. Fax (06) 2490740.

community is important. This is particularly so because there exists evidence that some of those attempting to alter their body weight may paradoxically put their health at risk. Many strategies to control body weight are available, ranging from dietary restriction and increased physical activity through to less desirable behaviours such as laxative and diuretic abuse, smoking for weight control, and extreme behaviours such as vomiting and purging. These strategies for weight control all have the potential, to a greater or lesser extent, to cause harm to health. ${ }^{4}$

Unsuccessful attempts to alter body weight (weight loss and subsequent regain) may also pose a significant long-term health risk. Large or frequent fluctuations in body weight may increase the risk of premature mortality, particularly from cardiovascular disease. ${ }^{5}$ Perhaps the most convincing evidence on the health effects of cycles of weight change is based on the 32-year follow-up data from the Framingham study. ${ }^{6}$ Multivariate analyses controlling for obesity, trends in weight over time, and indicators of cardiovascular disease revealed positive independent associations between weight 